\title{
Coronary Pulmonary Fistula: A Rare Case Presenting with Recurrent Chest Pain
}

Suvendu Mohapatra*, Jayashree mohanty and Basant Swain

Department of Radiology S.C.B Medical College, Cuttack, Odisha, India

*Corresponding author: Suvendu Mohapatra, Senior resident, Department of Radiology, S.C.B Medical College \& Hospital, Cuttack, Odisha, 753007, India, Tel: +09861412800; Fax: +916742390674, E-mail: drsuvendumohapatra@gmail.com

Received on: Mar 31, 2014, Accepted on: May 30, 2014, Published on: June 06, 2014

Copyright: () 2014 Mohapatra S et al. This is an open-access article distributed under the terms of the Creative Commons Attribution License, which permits unrestricted use, distribution, and reproduction in any medium, provided the original author and source are credited.

\begin{abstract}
Background: Coronary pulmonary fistula is a rare clinical entity. Majority of them are clinically silent. But rarely chest pain, myocardial ischaemia and heart failure may be the presenting features. Coronary artery fistulas originate predominantly from the right coronary artery and are not associated with hemodynamic abnormalities or other congenital heart diseases.
\end{abstract}

Case Presentation: Here we present a case of 41 years male presenting with recurrent chest pain for the last 2 years which had increased in frequency for last couple of days. Repeated Chest $x$ rays, electrocardiograms and echocardiography being normal. Lastly this case was diagnosed by CT coronary angiography which revealed normal caliber left and right coronary arteries but abnormal torturous plexus of vessels seen to arise from proximal part of left anterior descending artery finally communicating with pulmonary trunk suggestive of coronary pulmonary fistula.

Learning objective: Above case depicts an anomaly of coronary artery underdiagnosed since long time in many clinical scenarios in which patient might have repeated episodes of angina and this should be kept mind as a possible differential and CT angiography proves to be diagnostic in identifying the anomalous vessel with its origin and communications.

Keywords: Coronary; Fistula; Cardiac; Computerized Tomography; Angiography

\section{Introduction}

Coronary pulmonary fistulas are rare clinical entities most of the time being clinically silent. But rarely may they present as typical angina pectoris and heart failure in the absence of obstructive coronary arterial disease.

\section{Case Report}

We report a case of 41 years old Indian male, nonsmoker and nondiabetic presenting with recurrent central chest pain for the last 2 years. Recently he had increased severity and frequency of pain and association with strenuous activity. Multiple chest X rays, ECG and ultrasound abdomen are within normal limit. Blood pressure was also within normal limit (124/78 mm of $\mathrm{Hg}$ ). Other clinical examinations not revealing of any other associated anomaly. Family history was not suggestive of any cardiac illness or sudden death. Treadmill exercise showed the elevation of ST segment in lead I and aVL. Patient was reluctant for invasive angiography so was sent for CT coronary angiography. CT coronary angiography was performed in TOSHIBA AQUILION 128 slice volume CT scanner after injecting $100 \mathrm{cc}$ of nonionic contrast (Omnipaque 350) with power injector at speed $4 \mathrm{cc} /$ sec. Preprocedure beta blocker metoprolol $50 \mathrm{mg}$ was given before 1 hour to decrease the heart rate. Examination revealed there is no obstructive coronary pathology in right or left coronary arteries but an abnormal set of tortuous minimally dilated vessels seen originating from left anterior descending artery and its proximal part forming a vascular plexus ultimately communicating with main pulmonary trunk (Figure 1-3). It was concluded that patient's symptom was due to coronary blood shunting and possible coronary spasm.

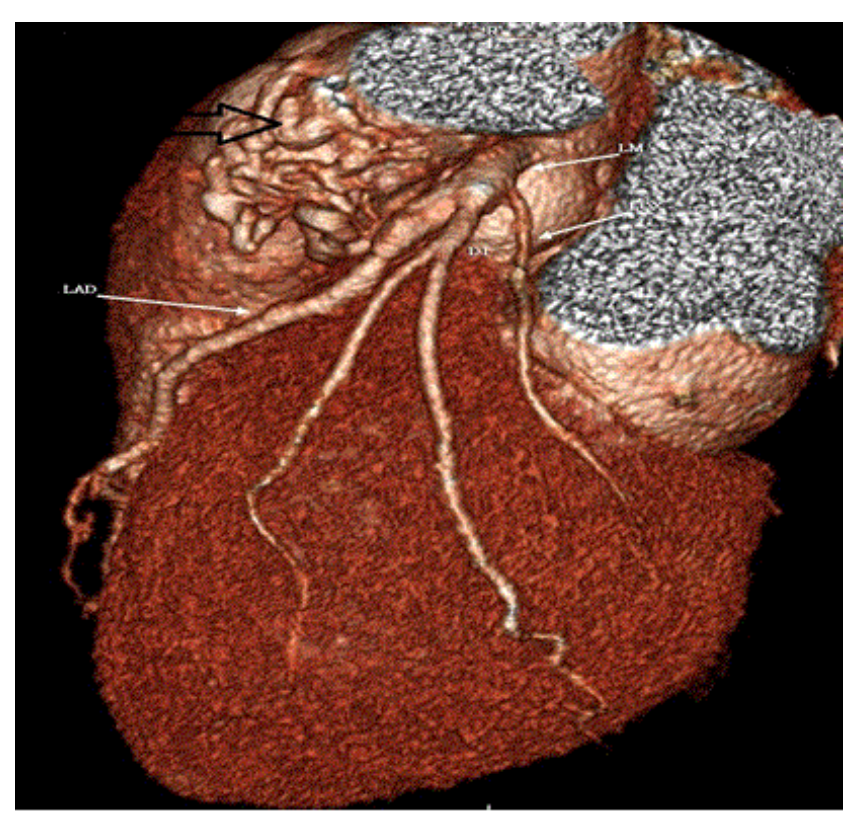

Figure 1: 3D coronary CT image reveal tortuous plexus of arteries from left anterior descending artery communicating with pulmonary trunk 
Page 2 of 2

Patient was reluctant to undergo intervention so was treated conservatively by the cardiologist. Patient was put on low dose anti platelet therapy and advised to undergo definitive therapy such as catheter-based closure of the fistulous connection or surgical correction of the anomaly. Patient remained asymptomatic on 6 month follow up.

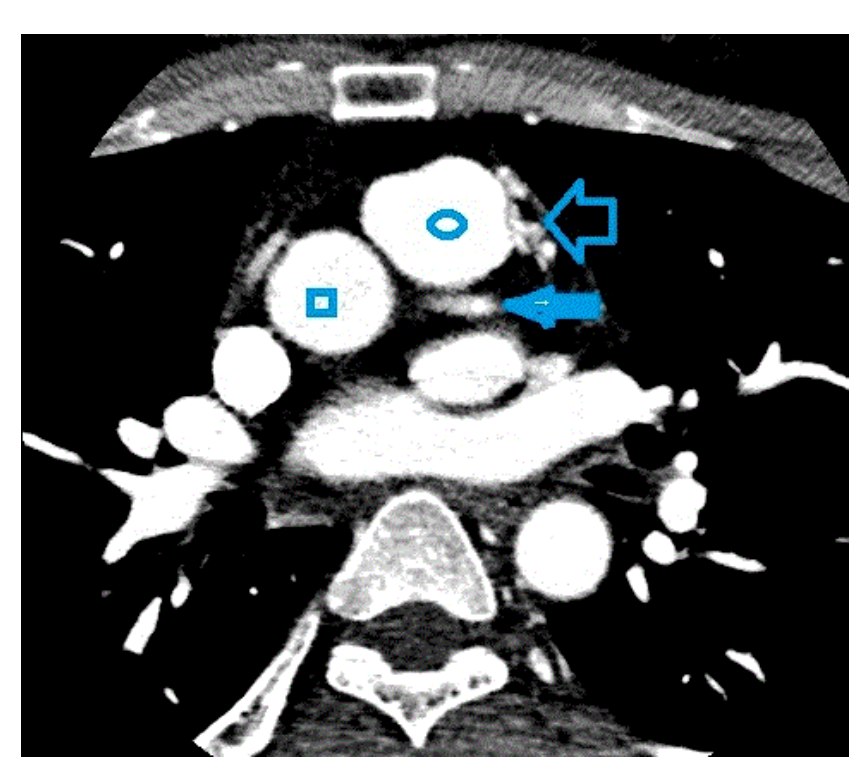

Figure 2: Axial Coronary CT reveals the abnormal vascular plexus (blue open arrow) with its opening to pulmonary trunk (blue circle). Left main coronary artery showed inferior to this (blue solid arrow). Ascending Aorta is shown by Blue Square

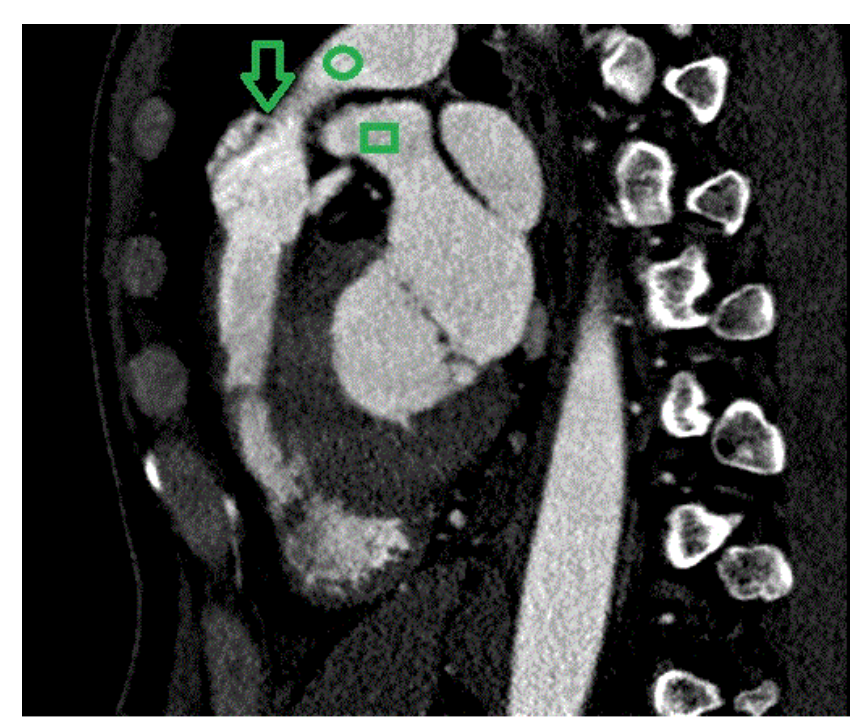

Figure 3: Sagittal coronary CT angiography reveal the abnormal vascular plexus opening to pulmonary trunk (point of communication shown in green arrow), pulmonary trunk shown by green circle. Aorta is shown by green square.

\section{Discussion}

Coronary pulmonary fistulas are mostly clinically silent. Coronarypulmonary fistulas are rare so rarely suspected in clinical scenarios as in our case 41 years male, a nonsmoker without any known risk factor for cardiac disease. On the basis of treadmill test showing ST segment elevation in lead I and aVL, patient underwent CT coronary angiography to look for any coronary obstruction but it revealed no significant calcific or noncalcific atherosclerotic load (calcium score of zero). However, left anterior descending artery showed an abnormal tortuous set of vessels forming a vascular plexus finally communicating with main pulmonary trunk. Coronary artery fistulas vary widely in their morphological appearance and presentation [1]. The incidence, angiographic characteristics and natural history of coronary artery fistulas in patients undergoing diagnostic cardiac catheterization have not been well defined [2]. The incidence of coronary artery fistulas detected during diagnostic coronary angiography is very low [2]. Coronary artery fistulas originate predominantly from the right coronary artery and are not associated with hemodynamic abnormalities or other congenital heart diseases [2]. Most of the patients are asymptomatic but heart failure, angina, myocardial infarction, endocarditis, and dyspnea have rarely been reported [3]. Although noninvasive imaging like CT coronary angiography may facilitate the diagnosis and identification of the origin and insertion of coronary artery fistulas, cardiac catheterization and coronary angiography is necessary for the precise delineation of coronary anatomy, for the assessment of hemodynamics, and to show the presence of concomitant atherosclerosis and other structural anomalies. Treatment is advocated for symptomatic patients and for those asymptomatic patients who are at risk for future complications. The management is controversial and recommendations are based on anecdotal cases or very small retrospective series. Possible therapeutic options include surgical correction and transcatheter embolization of the malformation.

\section{Conclusion}

Coronary arterial fistulas are rare malformation of coronary vessel. These are most often clinically silent. They may present with recurrent chest pain, cardiac ischaemia or cardiac failure. This disease is mostly underdiagnosed because of the rarity of this condition. Invasive coronary angiography is gold standard for both diagnosis and treatment of this condition but computerized tomographic coronary angiography can show the details of this malformation easily and noninvasively.

\section{References}

1. Gowda RM, Vasavada BC, Khan IA (2006) Coronary artery fistulas: clinical and therapeutic considerations. Int J Cardiol 107: 7-10.

2. Vavuranakis M, Bush CA, Boudoulas H (1995) Coronary artery fistulas in adults: incidence, angiographic characteristics, natural history. Cathet Cardiovasc Diagn 35: 116-120.

3. Said SA, Lam J, van der Werf T (2006) Solitary coronary artery fistulas: a congenital anomaly in children and adults. A contemporary review. Congenit Heart Dis 1: 63-76. 